\title{
RAMANUJAN'S REMARKABLE PRODUCT OF THETA-FUNCTIONS
}

\author{
by BRUCE C. BERNDT, HENG HUAT CHAN and LIANG-CHENG ZHANG
}

(Received 2nd February 1996)

Dedicated to Robert A. Rankin on the occasion of his 80 th birthday

\begin{abstract}
On pages 338 and 339 in his first notebook, Ramanujan records eighteen values for a certain product of theta-functions depending on two integral parameters $m$ and $n$. When $(m, n)=1$, it can be seen that each of these values is a unit. The purpose of this paper is to establish each of these eighteen values and to prove that under certain general conditions this product is indeed a unit. Lastly, we prove that certain quotients of theta-functions are algebraic integers.
\end{abstract}

1991 Mathematics subject classification: Primary, 33D10, 11F20, 11F27; Secondary, 33E05.

\section{Introduction}

As usual, set

$$
(a ; q)_{\infty}:=\prod_{n=0}^{\infty}\left(1-a q^{n}\right), \quad|q|<1 .
$$

After Ramanujan, define the theta-functions $\varphi(q)$ and $\psi(q)$ by

$$
\varphi(q):=\sum_{n=-\infty}^{\infty} q^{n^{2}}=\frac{(-q ;-q)_{\infty}}{(q ;-q)_{\infty}}
$$

and

$$
\psi(q):=\sum_{n=0}^{\infty} q^{n(n+1) / 2}=\frac{\left(q^{2} ; q^{2}\right)_{\infty}}{\left(q ; q^{2}\right)_{\infty}}
$$

where the infinite product representations arise from the Jacobi triple product identity $[1$, p. 36]. Also, the Dedekind eta-function $\eta(z)$ and Ramanujan's function $f(-q)$ are defined by

$$
\eta(z)=q^{1 / 24}(q ; q)_{\infty}=q^{1 / 24} f(-q), \quad q=e^{2 \times i z}, \operatorname{Im} z>0 .
$$

On page 338 in his first notebook [14], Ramanujan defines 


$$
a_{m, n}:=n e^{-(\pi / 4)(n-1) \sqrt{m / n}} \frac{\psi^{2}\left(e^{-\pi \sqrt{m n}}\right) \varphi^{2}\left(-e^{-2 \pi \sqrt{m n}}\right)}{\psi^{2}\left(e^{-\pi \sqrt{m / n}}\right) \varphi^{2}\left(-e^{-2 \pi \sqrt{m / n}}\right)},
$$

where evidently $m$ and $n$ are positive integers. He then, on pages 338 and 339 , offers a list of 18 particular values, which we present in the following table.

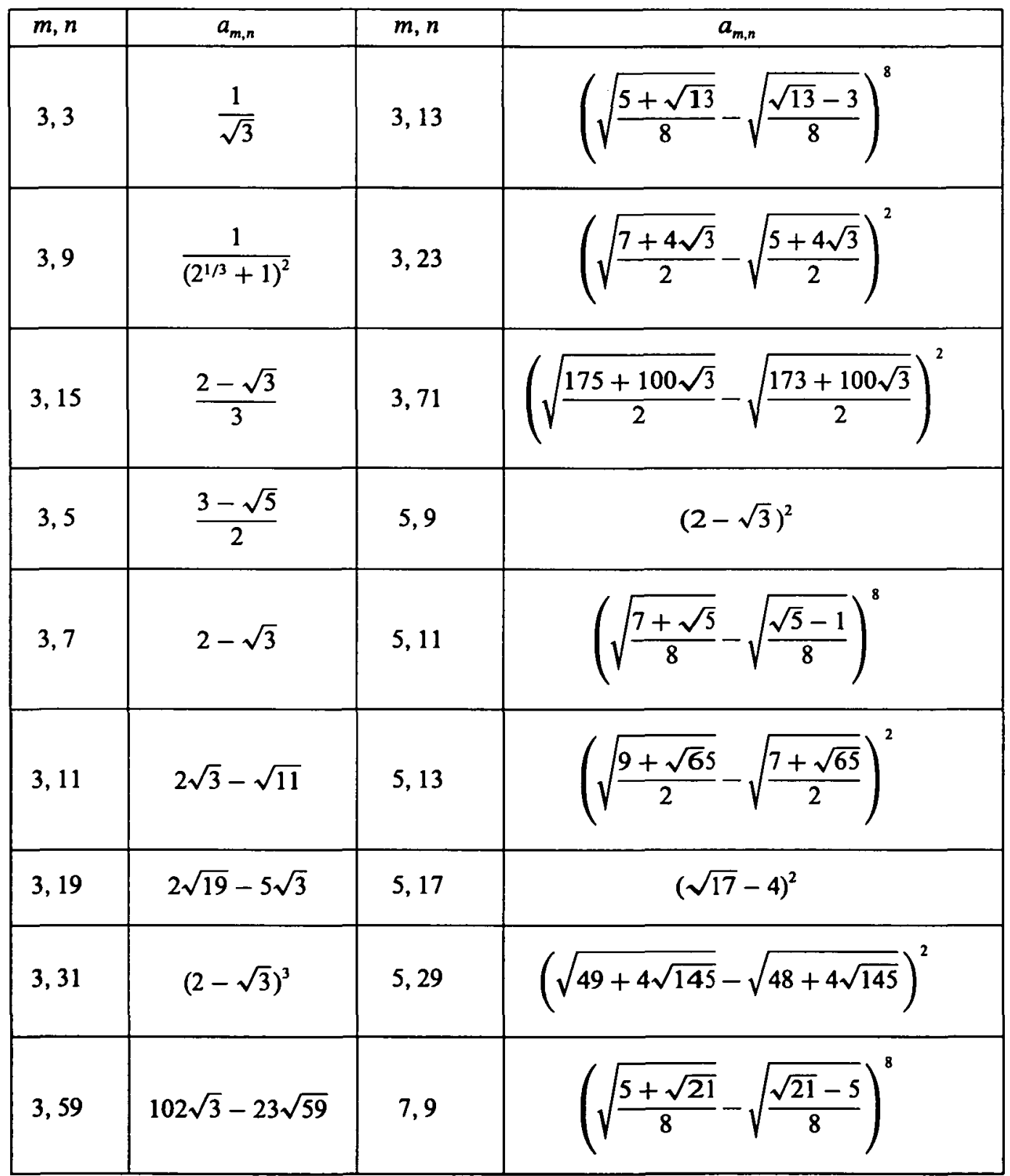


The first goal of this paper is to establish in Section 2 each of these eighteen values, and the tools we shall use were entirely known to Ramanujan. The methods we develop can be easily applied to determine further values of $a_{m, n}$, and we offer some of these. Upon examining the table, we see that when $(m, n)=1$ each value is a unit in some algebraic number field. Thus, our second goal in Section 3 is to prove that $a_{m, n}$ is a unit for large classes of pairs $m, n$. We also establish in Section 3 some general formulas for evaluating $a_{m, n}$. Here, we use ideal class theory, with which Ramanujan was likely unfamiliar. After stating his last value for $a_{m, n}$ on page 339, Ramanujan offers some values for $\varphi\left(e^{-n \pi}\right) / \varphi\left(e^{-n}\right)$, where $n$ is a positive integer. These values, and a few other values scattered throughout the first notebook, are proved in another paper [2]. Continuing in the spirit of Section 3, we prove in Section 4 that $\varphi\left(e^{-n \pi}\right) / \varphi\left(e^{-\pi}\right)$ is algebraic for every positive integer $n$.

Our calculations require primarily three of Ramanujan's modular equations, values for certain class invariants of Ramanujan, representations for quotients of values of $\varphi$ in terms of class invariants, and the theta-transformation formula.

We first recall the definition of a modular equation $\left[1\right.$, p. 213]. Let $K, K^{\prime}, L$, and $L^{\prime}$ denote complete elliptic integrals of the first kind associated with the moduli $k$, $k^{\prime}:=\sqrt{1-k^{2}}, \ell$, and $\ell^{\prime}:=\sqrt{1-\ell^{2}}$, respectively, where $0<k, \ell<1$. For a fixed positive integer $n$, suppose that

$$
n \frac{K^{\prime}}{K}=\frac{L^{\prime}}{L}
$$

Then a modular equation of degree $n$ is a relation between $k$ and $\ell$ induced by (1.5). Following Ramanujan, set $\alpha=k^{2}$ and $\beta=\ell^{2}$. We often say that $\beta$ has degree $n$ over $\alpha$. As customary in the theory of elliptic functions, set $q:=\exp \left(-\pi K^{\prime} / K\right)$.

Define

$$
\chi(q):=\left(-q ; q^{2}\right)_{\infty}
$$

For any positive rational number $r$, Ramanujan's class invariant $G_{r}$ is defined by

$$
G_{r}:=2^{-1 / 4} q^{-1 / 24} \chi(q),
$$

where $q=\exp (-\pi \sqrt{r})$. In the notation of Weber [17], $G_{r}=: 2^{-1 / 4} f(\sqrt{-r})$. It is wellknown that $G$, is algebraic; for example, see Cox's book [9, p. 214, Theorem 10.23; p. 257, Theorem 12.17]. Moreover, if $q=\exp (-\pi \sqrt{m / n})$ and $\beta$ has degree $n$ over $\alpha$, then $[1$, p. 124]

$$
G_{m / n}=\{4 \alpha(1-\alpha)\}^{-1 / 24} \quad \text { and } \quad G_{m n}=\{4 \beta(1-\beta)\}^{-1 / 24} .
$$

Next, we derive some formulas for $a_{m, n}$ that will be useful in Sections 2 and 3. Set $q=\exp (-\pi \sqrt{m / n})$. Then, by (1.1), (1.2), and (1.4), 


$$
a_{m, n}=n q^{(n-1) / 4} \frac{\left(q^{2 n} ; q^{2 n}\right)_{\infty}^{2}\left(q^{2 n} ; q^{2 n}\right)_{\infty}^{2}\left(q ; q^{2}\right)_{\infty}^{2}\left(-q^{2} ; q^{2}\right)_{\infty}^{2}}{\left(q^{n} ; q^{2 n}\right)_{\infty}^{2}\left(-q^{2 n} ; q^{2 n}\right)_{\infty}^{2}\left(q^{2} ; q^{2}\right)_{\infty}^{2}\left(q^{2} ; q^{2}\right)_{\infty}^{2}}
$$

Since $(-q ;-q)_{\infty}=\left(q^{2} ; q^{2}\right)_{\infty}\left(-q ;-q^{2}\right)_{\infty}$

$$
\begin{aligned}
\frac{\left(q^{2} ; q^{2}\right)_{\infty}^{2}}{\left(q ; q^{2}\right)_{\infty}\left(-q^{2} ;-q^{2}\right)_{\infty}} & =\frac{(-q ;-q)_{\infty}^{2}}{\left(q ; q^{2}\right)_{\infty}\left(-q^{2} ; q^{2}\right)_{\infty}\left(-q ; q^{2}\right)_{\infty}^{2}} \\
& =\frac{(-q ;-q)_{\infty}}{(q ;-q)_{\infty}\left(-q ; q^{2}\right)_{\infty}^{2}}
\end{aligned}
$$

Using Euler's identity,

$$
1=(q ;-q)_{\infty}\left(-q ; q^{2}\right)_{\infty}
$$

in (1.10) and then using (1.10) in (1.9) twice (once with $q$ replaced by $q^{n}$ ), we find that

$$
\begin{aligned}
a_{m, n} & =n q^{(n-1) / 4} \frac{\left(-q^{n} ;-q^{n}\right)_{\infty}^{4}(q ;-q)_{\infty}^{4}\left(-q ; q^{2}\right)_{\infty}^{6}}{\left(q^{n} ;-q^{n}\right)_{\infty}^{4}(-q ;-q)_{\infty}^{4}\left(-q^{n} ; q^{2 n}\right)_{\infty}^{6}} \\
& =n \frac{\varphi^{4}\left(e^{-\pi \sqrt{m n}}\right)}{\varphi^{4}\left(e^{-\pi \sqrt{m / n}}\right)} \frac{G_{m / n}^{6}}{G_{m n}^{6}}
\end{aligned}
$$

by (1.1) and (1.7). Using (1.11) once again in (1.10), but with the reverse substitution, and then using (1.10) in (1.9) twice, we also find that

$$
\begin{aligned}
a_{m, n} & =n q^{(n-1) / 4} \frac{\left(-q^{n} ;-q^{n}\right)_{\infty}^{4}\left(-q ; q^{2}\right)_{\infty}^{2}}{(-q ;-q)_{\infty}^{4}\left(-q^{n} ; q^{2 n}\right)_{\infty}^{2}} \\
& =n \frac{\eta^{4}\left(\frac{1+\sqrt{-m n}}{2}\right)}{\eta^{4}\left(\frac{1+\sqrt{-m / n}}{2}\right)} \frac{G_{m / n}^{2}}{G_{m n}^{2}}
\end{aligned}
$$

by (1.3) and (1.7). From (1.3) and (1.7), it is easy to see that, if $\tau=\sqrt{-r}$,

$$
G_{r}=2^{-1 / 4} \frac{\eta\left(\frac{1+\tau}{2}\right)}{\eta(\tau)}
$$

and so, using (1.14) in (1.13), we find that 


$$
a_{m, n}=n \frac{\eta^{2}\left(\frac{1+\sqrt{-m n}}{2}\right) \eta^{2}(\sqrt{-m n})}{\eta^{2}\left(\frac{1+\sqrt{-m / n}}{2}\right) \eta^{2}(\sqrt{-m / n})}
$$

K. G. Ramanathan [12, p. 88] very briefly discussed the numbers $a_{m, n}$ and derived an equivalent formulation of (1.13) (with a misprint). He wrote that he planned to return to $a_{m, n}$ in a future paper, but unfortunately he died before he was able to do so.

To help determine $G_{m / n}$, we shall employ three of Ramanujan's modular equations $[1$, pp. 231, 282, 315].

Lemma 1.1 (Modular Equation of Degree 3). Let

$$
P=\{16 \alpha \beta(1-\alpha)(1-\beta)\}^{1 / 8} \quad \text { and } \quad Q=\left(\frac{\beta(1-\beta)}{\alpha(1-\alpha)}\right)^{1 / 4}
$$

Then

$$
Q+\frac{1}{Q}+2 \sqrt{2}\left(P-\frac{1}{P}\right)=0
$$

Lemma 1.2 (Modular Equation of Degree 5). Let

$$
P=\{16 \alpha \beta(1-\alpha)(1-\beta)\}^{1 / 12} \quad \text { and } \quad Q=\left(\frac{\beta(1-\beta)}{\alpha(1-\alpha)}\right)^{1 / 8}
$$

Then

$$
Q+\frac{1}{Q}+2\left(P-\frac{1}{P}\right)=0
$$

Lemma 1.3 (Modular Equation of Degree 7). Let

$$
P=\{16 \alpha \beta(1-\alpha)(1-\beta)\}^{1 / 8} \quad \text { and } \quad Q=\left(\frac{\beta(1-\beta)}{\alpha(1-\alpha)}\right)^{1 / 6}
$$

Then

$$
Q+\frac{1}{Q}+7=2 \sqrt{2}\left(P+\frac{1}{P}\right)
$$

We also need three additional facts about $\varphi(q)$ and invariants. First, if $n$ is any 
positive rational number [6, p. 145], [2, eq. (3.10)],

$$
9 \frac{\varphi^{4}\left(e^{-3 \sqrt{n} \pi}\right)}{\varphi^{4}\left(e^{-\sqrt{n \pi}}\right)}=1+2 \sqrt{2} \frac{G_{9 n}^{3}}{G_{n}^{9}} .
$$

Second, if $\beta$ has degree 5 over $\alpha$ [1, p. 281, Entry 13(iv)],

$$
\begin{aligned}
5 \frac{\varphi^{2}\left(q^{5}\right)}{\varphi^{2}(q)} & =1+2^{4 / 3}\left(\frac{\alpha^{5}(1-\alpha)^{5}}{\beta(1-\beta)}\right)^{1 / 24} \\
& =1+2 \frac{G_{S_{n}}}{G_{n / 5}^{5}} \quad(\text { if } q=\exp (-\pi \sqrt{n / 5})),
\end{aligned}
$$

by (1.8). Third, we need the standard theta-transformation formula. If $\alpha, \beta>0$ with $\alpha \beta=\pi$, then [1, p. 43, Entry 27(i)]

$$
\sqrt{\alpha} \varphi\left(e^{-\alpha^{2}}\right)=\sqrt{\beta} \varphi\left(e^{-\beta^{2}}\right) .
$$

In particular, if $\alpha^{2}=\pi / \sqrt{r}$, then, from (1.21),

$$
\varphi\left(e^{-\pi / \sqrt{r}}\right)=r^{1 / 4} \varphi\left(e^{-\pi \sqrt{r}}\right)
$$

\section{Calculation of $a_{m, n}$}

\section{$2.1 m=3$}

We first calculate $a_{3,3}$. By (1.12),

$$
a_{3,3}=3 \frac{\varphi^{4}\left(e^{-3 \pi}\right)}{\varphi^{4}\left(e^{-\pi}\right)} \frac{G_{1}^{6}}{G_{9}^{6}} .
$$

Ramanujan recorded the value

$$
\frac{\varphi^{4}\left(e^{-3 \pi}\right)}{\varphi^{4}\left(e^{-n}\right)}=\frac{1}{6 \sqrt{3}-9}
$$

in his first notebook [14], and a proof can be found in [2, Theorem 2]. The values

$$
G_{1}=1 \quad \text { and } \quad G_{9}=\left(\frac{\sqrt{3}+1}{\sqrt{2}}\right)^{1 / 3}
$$


are well known [13], [15, p. 24], [17, p. 721]. Thus, putting these values in (2.1), we find that

$$
a_{3,3}=3 \frac{1}{6 \sqrt{3}-9}\left(\frac{\sqrt{2}}{\sqrt{3}+1}\right)^{2}=\frac{1}{\sqrt{3}}
$$

By (1.12) and (1.22),

$$
a_{3, n}=n \frac{\varphi^{4}\left(e^{-\pi \sqrt{3 n}}\right)}{\varphi^{4}\left(e^{-\pi \sqrt{3 / n}}\right)} \frac{G_{3 / n}^{6}}{G_{3 n}^{6}}=3 \frac{\varphi^{4}\left(e^{-\pi \sqrt{3 n}}\right)}{\varphi^{4}\left(e^{-\pi \sqrt{n / 3}}\right)} \frac{G_{n / 3}^{6}}{G_{3 n}^{6}},
$$

since $G_{n}=G_{1 / n}[13],[15$, p. 23]. Using (1.19) in (2.2), we find that

$$
a_{3, n}=\frac{1}{3}\left(\frac{G_{n / 3}^{6}}{G_{3 n}^{6}}+\frac{2 \sqrt{2}}{G_{n / 3}^{3} G_{3 n}^{3}}\right)
$$

Provided that the invariants $G_{n / 3}$ and $G_{3 n}$ are known, (2.3) can be utilized to compute several values of $a_{3, n}$.

First, let $n=9$. Then [17, pp. 721, 722], [14, vol. 1, pp. 282, 305, 345, 351; vol. 2, p. 294]

$$
G_{3}=2^{1 / 12} \quad \text { and } \quad G_{27}=2^{1 / 12}\left(2^{1 / 3}-1\right)^{-1 / 2}
$$

Putting these values in (2.3), upon simplification, we find that

$$
a_{3,9}=\frac{1}{3}\left(2^{2 / 3}-1\right)=\frac{1}{\left(2^{1 / 3}+1\right)^{2}}
$$

Second, let $n=15$. Then [17, pp. 721, 723], [14, vol. 1, p. 287; vol. 2, pp. 294, 295]

$$
G_{5}=\left(\frac{\sqrt{5}+1}{2}\right)^{1 / 4} \quad \text { and } \quad G_{45}=(\sqrt{5}+2)^{1 / 4}\left(\frac{\sqrt{5}+\sqrt{3}}{\sqrt{2}}\right)^{1 / 3}
$$

Using these values in (2.3), we find, after a straightforward, lengthy calculation, that

$$
a_{3,15}=\frac{2^{5 / 2}(1+2 \sqrt{3}+2 \sqrt{5})}{3(\sqrt{5}+\sqrt{3})^{2}(3+\sqrt{5})^{3 / 2}}
$$

But $\sqrt{3+\sqrt{5}}=\sqrt{5 / 2}+\sqrt{1 / 2}$. Thus, after further simplification, we find that 


$$
a_{3.15}=\frac{2(1+2 \sqrt{3}+2 \sqrt{5})}{3(\sqrt{5}+\sqrt{3})^{2}(2+\sqrt{5})}=\frac{2-\sqrt{3}}{3},
$$

by a direct calculation. (It is curious that the middle expression above is in $\mathbb{Q}(\sqrt{3})$ and not in $\mathbb{Q}(\sqrt{3}, \sqrt{5})$, as we would expect.)

In the remaining calculations, we need to calculate $G_{n / 3}$ when $n / 3$ is nonintegral. First, we could use the methods developed in [3], but these are nonelementary and depend upon the Kronecker limit formula. Second, with the use of (1.8), we can employ Lemma 1.1 with $P=\left(G_{n / 3} G_{3 n}\right)^{-3}$ and $Q=\left(G_{n / 3} / G_{3 n}\right)^{6}$ to deduce that

$$
\left(\frac{G_{n / 3}}{G_{3 n}}\right)^{6}+\left(\frac{G_{3 n}}{G_{n / 3}}\right)^{6}+2 \sqrt{2}\left(\frac{1}{G_{n / 3}^{3} G_{3 n}^{3}}+G_{n / 3}^{3} G_{3 n}^{3}\right)=0 .
$$

For specific values of $n$ and $G_{3 n}$, we could solve (2.5) for $G_{n / 3}$, but this procedure is normally very laborious. It is better to "guess" the solution and then to verify that our "guess" is indeed correct. That we have guessed the correct solution and not another solution can be simply verified by numerically checking all the roots of the polynomial equation.

Now set $n=5$ in (2.3). From Weber's book [17, p. 721] or Ramanujan's notebooks [14, vol. 1, p. 289; vol. 2, p. 294],

$$
G_{15}=2^{-1 / 12}(\sqrt{5}+1)^{1 / 3}
$$

From (2.5) we easily verify that

$$
G_{5 / 3}=2^{-1 / 12}(\sqrt{5}-1)^{1 / 3} .
$$

Using this value and (2.6) in (2.3), with $n=5$, we easily find that

$$
a_{3,5}=\frac{3-\sqrt{5}}{2} .
$$

Next, let $n=7$. From Weber's treatise $[17$, p. 722$]$ or Ramanujan's notebooks [14, vol. 1, p. 293; vol. 2, p. 294],

$$
G_{21}=2^{-1 / 3}(\sqrt{7}+\sqrt{3})^{1 / 4}(3+\sqrt{7})^{1 / 6} .
$$

Just as in the previous proof, we substitute this value in (2.5), when $n=7$, and verify that

$$
G_{7 / 3}=2^{-1 / 3}(\sqrt{7}-\sqrt{3})^{1 / 4}(3+\sqrt{7})^{1 / 6} .
$$

Using the foregoing values in (2.3) when $n=7$, we readily find that 


$$
a_{3.7}=2-\sqrt{3}
$$

Next, let $n=11$. From Ramanujan's notebooks [14, vol. 1, p. 311; vol. 2, p. 295] or from Weber's book [17, p. 722],

$$
G_{33}=2^{-1 / 3}(\sqrt{11}+3)^{1 / 6}(\sqrt{3}+1)^{1 / 2} .
$$

Letting $n=11$ in (2.5), we check that

$$
G_{11 / 3}=2^{-1 / 3}(\sqrt{11}-3)^{1 / 6}(\sqrt{3}+1)^{1 / 2}
$$

Upon putting these values in (2.3) when $n=11$, we readily deduce that

$$
a_{3,11}=2 \sqrt{3}-\sqrt{11}
$$

Let $n=19$. Either from Ramanujan's notebooks [14, vol. 1, p. 315; vol. 2, p. 295] or from Weber's book [17, p. 723],

$$
G_{57}=\left(\frac{3 \sqrt{19}+13}{\sqrt{2}}\right)^{1 / 6}(2+\sqrt{3})^{1 / 4}
$$

Putting $n=19$ in (2.5), we verify that

$$
G_{19 / 3}=\left(\frac{3 \sqrt{19}+13}{\sqrt{2}}\right)^{1 / 6}(2-\sqrt{3})^{1 / 4} .
$$

Employing these values in (2.3) with $n=19$, we easily deduce that

$$
a_{3,19}=2 \sqrt{19}-5 \sqrt{3}
$$

Let $n=31$. From either the notebooks [14, vol. 1, p. 315; vol. 2, p. 296] or Weber's treatise $[17$, p. 724$]$,

$$
G_{93}=\left(\frac{39+7 \sqrt{31}}{\sqrt{2}}\right)^{1 / 6}\left(\frac{\sqrt{31}+3 \sqrt{3}}{2}\right)^{1 / 4}
$$

Using (2.5) with $n=31$, we check that

$$
G_{31 / 3}=\left(\frac{39+7 \sqrt{31}}{\sqrt{2}}\right)^{1 / 6}\left(\frac{\sqrt{31}-3 \sqrt{3}}{2}\right)^{1 / 4} .
$$


Using these values in (2.3) when $n=31$, we easily find that

$$
a_{3,31}=(2-\sqrt{3})^{3} \text {. }
$$

Let $n=59$. From the notebooks [14, vol. 1, p. 315; vol. 2, p. 298] or Weber's book $[17$, p. 725$]$,

$$
G_{177}=\left(\frac{3 \sqrt{59}+23}{\sqrt{2}}\right)^{1 / 6}\left(\frac{\sqrt{3}+1}{\sqrt{2}}\right)^{3 / 2}
$$

By using (2.5) with $n=59$, we find that

$$
G_{59 / 3}=\left(\frac{3 \sqrt{59}-23}{\sqrt{2}}\right)^{1 / 6}\left(\frac{\sqrt{3}+1}{\sqrt{2}}\right)^{3 / 2} .
$$

With these values in (2.3) with $n=59$, it is now an easy task to show that

$$
a_{3,59}=102 \sqrt{3}-23 \sqrt{59}
$$

Let $n=39$. From Ramanujan's notebooks [14, vol. 1, p. 305; vol. 2, p. 295] or from Weber's book [17, p. 722],

$$
G_{39}=2^{1 / 4}\left(\frac{\sqrt{13}+3}{2}\right)^{1 / 6}\left(\sqrt{\frac{5+\sqrt{13}}{8}}+\sqrt{\frac{\sqrt{13}-3}{8}}\right)
$$

From (2.5), we can verify that

$$
G_{13 / 3}=2^{1 / 4}\left(\frac{\sqrt{13}+3}{2}\right)^{1 / 6}\left(\sqrt{\frac{5+\sqrt{13}}{8}}-\sqrt{\frac{\sqrt{13}-3}{8}}\right) .
$$

Using these values in (2.3) with $n=39$, after a calculation with the help of Mathematica, we find that

$$
\begin{aligned}
a_{3,13} & =\frac{1}{4}(7+3 \sqrt{13}-(4+\sqrt{13}) \sqrt{2 \sqrt{13}-2}) \\
& =\left(\sqrt{\frac{5+\sqrt{13}}{8}}-\sqrt{\frac{\sqrt{13}-3}{8}}\right)^{8},
\end{aligned}
$$

where we established the last equality by applying the binomial theorem on the right side. 
Let $n=23$. Although stated in his notebooks [14, vol. 1, pp. 314, 315; vol. 2, p. 295] and his paper [13], [15, p. 27], the value

$$
G_{69}=\left(\frac{5+\sqrt{23}}{\sqrt{2}}\right)^{1 / 12}\left(\frac{3 \sqrt{3}+\sqrt{23}}{2}\right)^{1 / 8}\left(\sqrt{\frac{6+3 \sqrt{3}}{4}}+\sqrt{\frac{2+3 \sqrt{3}}{4}}\right)^{1 / 2}
$$

was only recently established for the first time by the authors [3]. Next,

$$
G_{23 / 3}=\left(\frac{5-\sqrt{23}}{\sqrt{2}}\right)^{1 / 12}\left(\frac{3 \sqrt{3}-\sqrt{23}}{2}\right)^{1 / 8}\left(\sqrt{\frac{6+3 \sqrt{3}}{4}}+\sqrt{\frac{2+3 \sqrt{3}}{4}}\right)^{1 / 2}
$$

Once again, we can use (2.5) to verify this value, but the calculation is quite laborious. Alternatively, and preferably, we can use the method that we used in [3] to calculate $G_{69}$ to also calculate $G_{23 / 3}$. We use the values above in (2.3), when $n=23$, and find that, after a very lengthy calculation,

$$
\begin{aligned}
a_{3,23} & =6+4 \sqrt{3}-\frac{1}{2}(5 \sqrt{2}+3 \sqrt{6}) \sqrt{3 \sqrt{3}-2} \\
& =6+4 \sqrt{3}-\sqrt{(15 \sqrt{3}+26)(3 \sqrt{3}-2)} \\
& =\left(\sqrt{\frac{7+4 \sqrt{3}}{2}}-\sqrt{\frac{5+4 \sqrt{3}}{2}}\right)^{2} .
\end{aligned}
$$

Let $n=71$. In his notebooks [14, vol. 1, p. 315; vol. 2, p. 298] and paper [13], [15, p. 28], Ramanujan claimed that

$$
G_{213}=\left(\frac{5 \sqrt{3}+\sqrt{71}}{2}\right)^{1 / 8}\left(\frac{59+7 \sqrt{71}}{\sqrt{2}}\right)^{1 / 12}\left(\sqrt{\frac{21+12 \sqrt{3}}{2}}+\sqrt{\frac{19+12 \sqrt{3}}{2}}\right)^{1 / 2} .
$$

However, the authors [3] only recently gave the first proof. By using the same method that we employed in [3], which also utilized Lemma 1.1, or (2.5), we can deduce that

$$
G_{71 / 3}=\left(\frac{5 \sqrt{3}-\sqrt{71}}{2}\right)^{1 / 8}\left(\frac{59-7 \sqrt{71}}{\sqrt{2}}\right)^{1 / 12}\left(\sqrt{\frac{21+12 \sqrt{3}}{2}}+\sqrt{\frac{19+12 \sqrt{3}}{2}}\right)^{1 / 2} .
$$


Putting these two values in (2.3) when $n=71$, we find that, after a lengthy calculation,

$$
\begin{aligned}
a_{3,71} & =2(87+50 \sqrt{3})-\frac{1}{\sqrt{2}}(95+55 \sqrt{3}) \sqrt{5 \sqrt{3}-2} \\
& =2(87+50 \sqrt{3})-5 \sqrt{(362+209 \sqrt{3})(5 \sqrt{3}-2)} \\
& =\left(\sqrt{\frac{175+100 \sqrt{3}}{2}}-\sqrt{\frac{173+100 \sqrt{3}}{2}}\right)^{2}
\end{aligned}
$$

We close this subsection by proving two additional formulas for $a_{3, n}$. Theorem 2.1 below is an analogue of Corollary 2.4 (for $m=5$ ) and Theorem 2.5 (for $m=7$ ), and provides an optional method for calculating $a_{3, n}$. For calculational purposes, the formulas for $m=5,7$ are more advantageous than the ones below for $m=3$.

Theorem 2.1. If $n$ is a positive integer and

$$
V_{n}=\frac{G_{n / 3}}{G_{3 n}}
$$

then

$$
a_{3, n}-\frac{1}{a_{3, n}}=\frac{1}{3}\left(V_{n}^{6}-V_{n}^{-6}\right)
$$

Proof. If $\beta$ has degree 3 over $\alpha$, then [1, p. 230, Entry 5(vii)]

$$
m^{2}=\left(\frac{\beta}{\alpha}\right)^{1 / 2}+\left(\frac{1-\beta}{1-\alpha}\right)^{1 / 2}-\left(\frac{\beta(1-\beta)}{\alpha(1-\alpha)}\right)^{1 / 2}
$$

and

$$
\frac{9}{m^{2}}=\left(\frac{\alpha}{\beta}\right)^{1 / 2}+\left(\frac{1-\alpha}{1-\beta}\right)^{1 / 2}-\left(\frac{\alpha(1-\alpha)}{\beta(1-\beta)}\right)^{1 / 2}
$$

where $m=\varphi^{2}(q) / \varphi^{2}\left(q^{3}\right)$. By combining (2.8) and (2.9), we deduce that

$$
\left(\frac{\alpha(1-\alpha)}{\beta(1-\beta)}\right)^{1 / 2}\left(m^{2}+\left(\frac{\beta(1-\beta)}{\alpha(1-\alpha)}\right)^{1 / 2}\right)=\frac{9}{m^{2}}+\left(\frac{\alpha(1-\alpha)}{\beta(1-\beta)}\right)^{1 / 2}
$$


Let $q=\exp (-\pi \sqrt{n / 3})$, so that $q^{3}=\exp (-\pi \sqrt{3 n})$. Then, by $(1.8), G_{n / 3}=\{4 \alpha(1-\alpha)\}^{-1 / 24}$ and $G_{3 n}=\{4 \beta(1-\beta)\}^{-1 / 24}$. Thus, (2.10) may be written in the form

$$
\left(\frac{G_{3 n}}{G_{n / 3}}\right)^{12}\left(m^{2}+\left(\frac{G_{n / 3}}{G_{3 n}}\right)^{12}\right)=\frac{9}{m^{2}}+\left(\frac{G_{3 n}}{G_{n / 3}}\right)^{12} .
$$

Rearranging (2.11) and using (1.12), we readily deduce (2.7).

By combining Theorem 2.1 with the modular equation (2.5), it is not difficult to deduce the following corollary.

Corollary 2.2. If $n$ is a positive integer and $U_{n}=G_{n / 3} G_{3 n}$, then

$$
a_{3, n}-\frac{1}{a_{3, n}}=\frac{1}{3}\left(8 U_{n}^{6}+8 U_{n}^{-6}-20\right)^{1 / 2}
$$

\section{$2.2 m=5$}

First, let $n=9$. By (1.12) and two applications of (1.19),

$$
\begin{aligned}
a_{5,9} & =9 \frac{\varphi^{4}\left(e^{-\pi \sqrt{45}}\right)}{\varphi^{4}\left(e^{-\pi \sqrt{5 / 9}}\right)} \frac{G_{5 / 9}^{6}}{G_{45}^{6}} \\
& =9 \frac{\varphi^{4}\left(e^{-\pi \sqrt{45}}\right)}{\varphi^{4}\left(e^{-\pi \sqrt{5}}\right)} \frac{\varphi^{4}\left(e^{-\pi \sqrt{5}}\right)}{\varphi^{4}\left(e^{-\pi \sqrt{5} / 3}\right)} \frac{G_{9 / 5}^{6}}{G_{45}^{6}} \\
& =\frac{1}{9}\left(1+2 \sqrt{2} \frac{G_{45}^{3}}{G_{5}^{9}}\right)\left(1+2 \sqrt{2} \frac{G_{5}^{3}}{G_{5 / 9}^{9}}\right) \frac{G_{9 / 5}^{6}}{G_{45}^{6}} .
\end{aligned}
$$

Next, by (1.8) and Lemma 1.2 with $P=\left(G_{n / 5} G_{5 n}\right)^{-2}$ and $Q=\left(G_{n / 5} / G_{5 n}\right)^{3}$,

$$
\left(\frac{G_{n / 5}}{G_{5 n}}\right)^{3}+\left(\frac{G_{5 n}}{G_{n / 5}}\right)^{3}+2\left(\frac{1}{G_{n / 5}^{2} G_{5 n}^{2}}-G_{n / 5}^{2} G_{5 n}^{2}\right)=0 .
$$

Set $n=9$ in (2.13) and substitute the value of $G_{45}$ given by (2.4). We then verify that, as in our applications of (2.5),

$$
G_{9 / 5}=(\sqrt{5}+2)^{1 / 4}\left(\frac{\sqrt{5}-\sqrt{3}}{\sqrt{2}}\right)^{1 / 3} .
$$

Putting this value and the values for $G_{5}$ and $G_{45}$, given by (2.4), into (2.12), we find, after a long, but straightforward, calculation, that 


$$
a_{5,9}=(2-\sqrt{3})^{2}
$$

From (1.12) and (1.20),

$$
a_{5, n}=5 \frac{\varphi^{4}\left(e^{-\pi \sqrt{5 n}}\right)}{\varphi^{4}\left(e^{-\pi \sqrt{n / 5}}\right)} \frac{G_{n / 5}^{6}}{G_{5 n}^{6}}=\frac{1}{5}\left(1+2 \frac{G_{5 n}}{G_{n / 5}^{5}}\right)^{2} \frac{G_{n / 5}^{6}}{G_{5 n}^{6}} .
$$

We shall use (2.14) in further computations and in our proof of Corollary 2.4 below.

We next derive a formula by which the computation of $a_{5, n}$ generally will be simpler than that by using an analogue of (2.3). The following theorem is proved in our paper [4].

Theorem 2.3. Let $f(-q)$ be defined by (1.3), and let $k$ be a positive rational number. Put

$$
A_{1}^{\prime}=e^{\pi \sqrt{k} / 6} \frac{f\left(e^{-\pi \sqrt{k}}\right)}{f\left(e^{-5 \pi \sqrt{k}}\right)} \quad \text { and } \quad V^{\prime}=\frac{G_{25 k}}{G_{k}}
$$

Then

$$
\frac{A_{1}^{\prime 2} V^{\prime}}{\sqrt{5}}-\frac{\sqrt{5}}{A_{1}^{\prime 2} V^{\prime}}=\frac{1}{\sqrt{5}}\left(V^{\prime 3}-V^{\prime-3}\right)
$$

Corollary 2.4. If $n$ is a positive integer and

$$
V_{n}=\frac{G_{n / 5}}{G_{5 n}}
$$

then

$$
\begin{aligned}
\sqrt{a_{5, n}}-\frac{1}{\sqrt{a_{5, n}}} & =\frac{1}{\sqrt{5}}\left(V_{n}^{3}-V_{n}^{-3}\right) \\
& =\frac{1}{\sqrt{5}}\left(V_{n}-V_{n}^{-1}\right)\left(\left(V_{n}-V_{n}^{-1}\right)^{2}+3\right)
\end{aligned}
$$

Proof. From (2.14) and (1.22),

$$
a_{5, n}=\frac{1}{5} \frac{\varphi^{4}\left(e^{-\pi / \sqrt{5 n}}\right)}{\varphi^{4}\left(e^{-\pi \sqrt{5 / n}}\right)} \frac{G_{5 / n}^{6}}{G_{1 /(5 n)}^{6}}
$$


and from (1.1), (1.3), and (1.6), with $k=1 /(5 n)$ in the definition of $A_{1}^{\prime}$,

$$
A_{1}^{\prime}=e^{\pi /(6 \sqrt{5 n})} \frac{\varphi\left(e^{-\pi / \sqrt{5 n}}\right)}{\varphi\left(e^{-\pi \sqrt{5 / n}}\right)} \frac{\chi\left(e^{-\pi \sqrt{5 / n}}\right)}{\chi\left(e^{-\pi / \sqrt{5 n}}\right)} .
$$

Therefore,

$$
\frac{A_{1}^{2} V^{\prime}}{\sqrt{5}}=\frac{1}{\sqrt{5}} \frac{\varphi^{2}\left(e^{-\pi / \sqrt{5 n}}\right)}{\varphi^{2}\left(e^{-\pi \sqrt{5 / n}}\right)} \frac{G_{5 / n}^{2}}{G_{1 /(5 n)}^{2}} \frac{G_{5 / n}}{G_{1 /(5 n)}}=\sqrt{a_{5, n}} .
$$

Thus, by Theorem 2.3,

$$
\sqrt{a_{5, n}}-\frac{1}{\sqrt{a_{5, n}}}=\frac{1}{\sqrt{5}}\left(V_{n}^{3}-V_{n}^{-3}\right)
$$

since $V^{\prime}=V_{n}$. This completes the proof.

Now let $n=11$. From Ramanujan's notebooks [14, vol. 1, p. 315; vol. 2, p. 295] or Weber's treatise [17, p. 723],

$$
G_{55}=2^{1 / 4}(\sqrt{5}+2)^{1 / 6}\left(\sqrt{\frac{7+\sqrt{5}}{8}}+\sqrt{\frac{\sqrt{5}-1}{8}}\right) .
$$

Setting $n=11$ in (2.13), we verify that

$$
G_{11 / 5}=2^{1 / 4}(\sqrt{5}+2)^{1 / 6}\left(\sqrt{\frac{7+\sqrt{5}}{8}}-\sqrt{\frac{\sqrt{5}-1}{8}}\right) .
$$

Thus,

$$
V_{11}-V_{11}^{-1}=-\sqrt{\frac{3 \sqrt{5}-1}{2}}
$$

and so from Corollary 2.4 ,

$$
\sqrt{a_{5,11}}-\frac{1}{\sqrt{a_{5,11}}}=-\frac{1}{\sqrt{5}} \sqrt{\frac{3 \sqrt{5}-1}{2}}\left(\frac{3 \sqrt{5}+5}{2}\right)=-\sqrt{\frac{19+9 \sqrt{5}}{2}}
$$

Solving for $\sqrt{a_{5,11}}$, we find that 


$$
\begin{aligned}
a_{5,11} & =\left(\sqrt{\frac{27+9 \sqrt{5}}{8}}-\sqrt{\frac{19+9 \sqrt{5}}{8}}\right)^{2} \\
& =\left(\sqrt{\frac{7+\sqrt{5}}{8}}-\sqrt{\frac{\sqrt{5}-1}{8}}\right)^{8} .
\end{aligned}
$$

Let $n=13$. The value of $G_{65}$ is given by Ramanujan in his notebooks $[14$, vol. $1, \mathrm{p}$. 315 ; vol. 2, p. 295] and paper [13], [15, p. 27], but it was only recently established for the first time by the authors [3]. From [3, Section 7],

$$
V_{13}=\sqrt{\frac{\sqrt{65}+7}{8}}-\sqrt{\frac{\sqrt{65}-1}{8}}
$$

Thus,

$$
V_{13}-V_{13}^{-1}=-\sqrt{\frac{\sqrt{65}-1}{2}}
$$

Therefore, by Corollary 2.4 ,

$$
\sqrt{a_{5,13}}-\frac{1}{\sqrt{a_{5,13}}}=-\frac{5+\sqrt{65}}{2 \sqrt{5}} \sqrt{\frac{\sqrt{65}-1}{2}}=-\sqrt{14+2 \sqrt{65}}
$$

Hence,

$$
\sqrt{a_{5,13}}+\frac{1}{\sqrt{a_{5,13}}}=\sqrt{18+2 \sqrt{65}}
$$

Solving for $a_{5,13}$, we deduce that

$$
a_{5,13}=\left(\sqrt{\frac{9+\sqrt{65}}{2}}-\sqrt{\frac{7+\sqrt{65}}{2}}\right)^{2}
$$

Let $n=17$. From Ramanujan's notebooks [14, vol. 1, p. 315; vol. 2, p. 296] or paper [13], [15, p. 26], or from Weber's treatise [17, p. 724],

$$
G_{85}=\left(\frac{\sqrt{5}+1}{2}\right)\left(\frac{\sqrt{85}+9}{2}\right)^{1 / 4}
$$


With $n=17$ in (2.13), it is quite easy to verify that

$$
G_{17 / 5}=\left(\frac{\sqrt{5}-1}{2}\right)\left(\frac{\sqrt{85}+9}{2}\right)^{1 / 4}
$$

Putting these values in (2.14), with $n=17$, we readily find that

$$
a_{5,17}=(\sqrt{17}-4)^{2}
$$

Next, let $n=29$. In his notebooks [14, vol. 1, p. 315; vol. 2, p. 298] and paper [13], [15, p. 28], Ramanujan gave the value for $G_{145}$, which was proved for the first time by the authors in [3]. In the course of establishing this value, we proved that [3, Section 7]

$$
V_{29}=\sqrt{\frac{17+\sqrt{145}}{8}}-\sqrt{\frac{9+\sqrt{145}}{8}}
$$

Thus,

$$
V_{29}-V_{29}^{-1}=-\sqrt{\frac{9+\sqrt{145}}{2}}
$$

and so, by Corollary 2.4 ,

$$
\sqrt{a_{5,29}}-\frac{1}{\sqrt{a_{5,29}}}=-\frac{15+\sqrt{145}}{2 \sqrt{5}} \sqrt{\frac{9+\sqrt{145}}{2}}=-\sqrt{192+16 \sqrt{145}}
$$

Hence,

$$
\sqrt{a_{5,29}}+\frac{1}{\sqrt{a_{5,29}}}=\sqrt{196+16 \sqrt{145}}
$$

Solving for $a_{5,29}$, we conclude that

$$
a_{5,29}=(\sqrt{49+4 \sqrt{145}}-\sqrt{48+4 \sqrt{145}})^{2}
$$

Similarly, by using Theorems $4.6,4.8,4.10$, and 4.11 of [3], we can deduce the values 


$$
\begin{aligned}
& a_{5,41}=\left(\sqrt{\frac{23+3 \sqrt{41}}{4}}-\sqrt{\frac{19+3 \sqrt{41}}{4}}\right)^{4}, \\
& a_{5,53}=\left(\sqrt{\frac{5 \sqrt{53}+17 \sqrt{5}+2}{4}}-\sqrt{\frac{5 \sqrt{53}+17 \sqrt{5}-2}{4}}\right)^{4}, \\
& a_{5,89}=\left(\sqrt{\frac{85+9 \sqrt{89}}{2}}-\sqrt{\frac{83+9 \sqrt{89}}{2}}\right)^{4},
\end{aligned}
$$

and

$$
a_{5,101}=\left(\sqrt{\frac{13 \sqrt{101}+58 \sqrt{5}+1}{2}}-\sqrt{\frac{13 \sqrt{101}+58 \sqrt{5}-1}{2}}\right)^{4} .
$$

$2.3 m=7$

First, let $n=9$. By (1.12) and two applications of (1.19),

$$
\begin{aligned}
a_{7,9} & =9 \frac{\varphi^{4}\left(e^{-3 \pi \sqrt{7}}\right)}{\varphi^{4}\left(e^{-\pi \sqrt{7}}\right)} \frac{\varphi^{4}\left(e^{-\pi \sqrt{7}}\right)}{\varphi^{4}\left(e^{-\pi \sqrt{7} / 3}\right)} \frac{G_{9 / 7}^{6}}{G_{63}^{6}} \\
& =\frac{1}{9}\left(1+2 \sqrt{2} \frac{G_{63}^{3}}{G_{7}^{9}}\right)\left(1+2 \sqrt{2} \frac{G_{7}^{3}}{G_{7 / 9}^{9}}\right) \frac{G_{9 / 7}^{6}}{G_{63}^{6}} .
\end{aligned}
$$

Using (1.8) and setting $P=\left(G_{n / 7} G_{7 n}\right)^{-3}$ and $Q=\left(G_{n / 7} / G_{7 n}\right)^{4}$, we find that (1.18) in Lemma 1.3 assumes the form

$$
\left(\frac{G_{n / 7}}{G_{7 n}}\right)^{4}+\left(\frac{G_{7 n}}{G_{n / 7}}\right)^{4}+7=2 \sqrt{2}\left(\frac{1}{G_{n / 7}^{3} G_{7 n}^{3}}-G_{n / 7}^{3} G_{7 n}^{3}\right) .
$$

From either Ramanujan's notebooks [14, vol. 1, p. 305; vol. 2, p. 295] or Weber's treatise [17, p. 723],

$$
G_{63}=2^{1 / 4}\left(\frac{5+\sqrt{21}}{2}\right)^{1 / 6}\left(\sqrt{\frac{5+\sqrt{21}}{8}}+\sqrt{\frac{\sqrt{21}-3}{8}}\right) .
$$

Using (2.16) with $n=9$, we easily verify that 


$$
G_{9 / 7}=2^{1 / 4}\left(\frac{5+\sqrt{21}}{2}\right)^{1 / 6}\left(\sqrt{\frac{5+\sqrt{21}}{8}}-\sqrt{\frac{\sqrt{21}-3}{8}}\right)
$$

Substituting in (2.15) and using the denestings

$$
\sqrt{5 \pm \sqrt{21}}=\sqrt{7 / 2} \pm \sqrt{3 / 2}
$$

we find that

$$
\begin{aligned}
a_{7,9} & =\frac{1}{8}(62+14 \sqrt{21}-(21 \sqrt{6}+13 \sqrt{14}) \sqrt{\sqrt{21}-3}) \\
& =\left(\sqrt{\frac{5+\sqrt{21}}{8}}-\sqrt{\frac{\sqrt{21}-3}{8}}\right)
\end{aligned}
$$

upon expanding the right side and simplifying.

Of course, the method of calculation used above cannot be generalized to $n \neq 9$. Thus, generally, it seems best to prove an analogue of Theorem 2.1 and Corollary 2.4 .

Theorem 2.5. If $n$ is any positive integer and

$$
V_{n}=\frac{G_{n / 7}}{G_{7 n}}
$$

then

$$
\begin{aligned}
a_{7, n}-\frac{1}{a_{7, n}} & =\frac{1}{7}\left(\left(V_{n}^{6}-V_{n}^{-6}\right)+8\left(V_{n}^{2}-V_{n}^{-2}\right)\right) \\
& =\frac{1}{7}\left(V_{n}^{2}-V_{n}^{-2}\right)\left(\left(V_{n}^{2}-V_{n}^{-2}\right)^{2}+11\right) .
\end{aligned}
$$

The proof of Theorem 2.5 follows along exactly the same lines as that for Theorem 2.1. The relevant modular equations of degree 7 are given by $[1$, p. 314, Entry $9(v)]$

$$
m^{2}=\left(\frac{\beta}{\alpha}\right)^{1 / 2}+\left(\frac{1-\beta}{1-\alpha}\right)^{1 / 2}-\left(\frac{\beta(1-\beta)}{\alpha(1-\alpha)}\right)^{1 / 2}-8\left(\frac{\beta(1-\beta)}{\alpha(1-\alpha)}\right)^{1 / 3}
$$

and 


$$
\frac{49}{m^{2}}=\left(\frac{\alpha}{\beta}\right)^{1 / 2}+\left(\frac{1-\alpha}{1-\beta}\right)^{1 / 2}-\left(\frac{\alpha(1-\alpha)}{\beta(1-\beta)}\right)^{1 / 2}-8\left(\frac{\alpha(1-\alpha)}{\beta(1-\beta)}\right)^{1 / 3},
$$

where $m=\varphi^{2}(q) / \varphi^{2}\left(q^{7}\right)$.

As an example, let $n=11$. Then from [3, Section 7],

$$
V_{11}^{2}=\sqrt{\frac{23+8 \sqrt{11}}{4}}-\sqrt{\frac{19+8 \sqrt{11}}{4}} .
$$

Hence,

$$
V_{11}^{2}-V_{11}^{-2}=-\sqrt{19+8 \sqrt{11}}
$$

and, from Theorem 2.5,

$$
a_{7,11}-\frac{1}{a_{7,11}}=-\frac{30+8 \sqrt{11}}{7} \sqrt{19+8 \sqrt{11}}=-\sqrt{1484+448 \sqrt{11}} .
$$

Solving for $a_{7,11}$, we deduce that

$$
a_{7,11}=\sqrt{372+112 \sqrt{11}}-\sqrt{371+112 \sqrt{11}}
$$

\section{3. $a_{m, n}$ and units}

Some of the introductory material in this section arises from H. M. Stark's paper [16], while the proofs later in the section are heavily dependent on results in our paper [3].

Set

$$
b_{m, n}=\sqrt{n} \frac{\eta^{2}\left(\frac{1+\sqrt{-m n}}{2}\right)}{\eta^{2}\left(\frac{1+\sqrt{-m / n}}{2}\right)} \quad \text { and } \quad c_{m, n}=\sqrt{n} \frac{\eta^{2}(\sqrt{-m n})}{\eta^{2}(\sqrt{-m / n})}
$$

so that, by (1.15),

$$
a_{m, n}=b_{m, n} c_{m, n}
$$

Recall that the discriminant function $\Delta(z)$ is defined by

$$
\Delta(z)=\eta^{24}(z)
$$


It is well known that $\Delta(z)$ is a modular form of weight 12 on the full modular group $\Gamma$ with multiplier system identically equal to 1 , i.e., if $A=\left[\begin{array}{ll}a & b \\ c & d\end{array}\right] \in \Gamma$, then

$$
\Delta(A z)=(c z+d)^{12} \Delta(z)
$$

By (3.1),

$$
b_{m, n}^{24}=n^{12} \frac{\Delta^{2}\left(\frac{1+\sqrt{-m n}}{2}\right)}{\Delta^{2}\left(\frac{1+\sqrt{-m / n}}{2}\right)} \quad \text { and } \quad c_{m, n}^{24}=n^{12} \frac{\Delta^{2}(\sqrt{-m n})}{\Delta^{2}(\sqrt{-m / n})}
$$

For complex numbers $z_{1}, z_{2}$ with $\operatorname{Im}\left(z_{1} / z_{2}\right)>0$, define

$$
\Delta\left(\begin{array}{l}
z_{1} \\
z_{2}
\end{array}\right)=z_{2}^{-12} \Delta(z)
$$

where $z=z_{1} / z_{2}$. A brief calculation, utilizing (3.3) and (3.5), shows that

$$
\Delta\left(A\left[\begin{array}{l}
z_{1} \\
z_{2}
\end{array}\right]\right)=\Delta\left(\begin{array}{l}
z_{1} \\
z_{2}
\end{array}\right)
$$

Let $K=Q(\sqrt{-d})$, where $d$ is a positive squaref ree integer. Set

$$
\Omega=\left\{\begin{aligned}
\sqrt{-d}, & \text { if }-d \equiv 2,3(\bmod 4), \\
\frac{1+\sqrt{-d}}{2}, & \text { if }-d \equiv 1(\bmod 4) .
\end{aligned}\right.
$$

The ring of algebraic integers in $K$ is $\mathbb{Z}[\Omega]$. It is well known that any ideal of $K$ can be expressed as a $\mathbb{Z}$-module of the form $I:=[b+c \Omega, a]$, with $a, c>0, c|b, c| a$, and $a \mid N(b+\Omega)$, where $N(\gamma)$ denotes the norm of an algebraic integer $\gamma$. The ideal $I$ is primitive if $c=1$. It is also well known that there is a one-to-one correspondence between primitive ideals of $K$ and primitive positive definite binary quadratic forms of discriminant equal to $-\sigma^{2} d$, where $\sigma=1$ if $-d \equiv 1(\bmod 4)$, and $\sigma=2$ if $-d \equiv 2,3(\bmod 4)$. In fact, for $I=[b+\Omega, a]$, the corresponding primitive form is given by $Q(x, y):=a(x+y w)(x+y \bar{w})$, where $w=(b+\Omega) / a$. On the other hand, if $Q(x, y)=a x^{2}+2 b x y+c y^{2}$, where $4\left(b^{2}-a c\right)=-\sigma^{2} d$, the corresponding primitive ideal is $[b+\Omega, a]$.

Let

$$
S_{1}(f)=\left\{A=\left[\begin{array}{ll}
r & s \\
t & u
\end{array}\right]: r, s, t, u \in \mathbb{Z}, \operatorname{gcd}(r, s, t, u)=1, \operatorname{det} A=f\right\}
$$

For $z=z_{1} / z_{2}$, with $\operatorname{Im} z>0$, and $A \in S_{1}(f)$, define 


$$
g_{A}(z):=f^{12} \frac{\Delta\left(A\left[\begin{array}{l}
z_{1} \\
z_{2}
\end{array}\right]\right)}{\Delta\left(\begin{array}{l}
z_{1} \\
z_{2}
\end{array}\right)}
$$

The following lemma is a restatement in terms of ideals of a lemma in Stark's paper [16, Lemma 16].

Lemma 3.1. Let $I_{1}=[b+\Omega, a]$ and $I_{2}=\left[b^{\prime}+\Omega, a^{\prime}\right]$ be two primitive ideals. Let $A \in S_{1}(f)$, such that $I_{1} A=I_{2}$, where $I_{1}$ and $I_{2}$ are formally considered here to be row matrices. Then

$$
f^{-12}\left|g_{A}(w)\right|^{2}
$$

is a unit, where $w=(b+\Omega) / a$, and where $g_{A}(z)$ is defined in (3.8).

By equating like entries in the equation $I_{1} A=I_{2}$, we find that

$$
f=a^{\prime} / a \text {. }
$$

Thus, by Lemma 3.1,

$$
\frac{a^{12}}{a^{\prime 2}}\left|g_{A}\left(\frac{b+\Omega}{a}\right)\right|^{2}
$$

is a unit.

With the help of Lemma 3.1, we study $a_{m, n}$.

Theorem 3.2. Let $m$ and $n$ be odd positive integers such that $m n$ is squarefree and $-m n \equiv 3(\bmod 4)$. Then $a_{m, n}$ is a unit. Proof. Apply Lemma 3.1 with $I_{1}=[\Omega, 1]$ and $I_{2}=[\Omega, n]$. Then $A=\left[\begin{array}{ll}1 & 0 \\ 0 & n\end{array}\right]$ and,
by (3.9), $f=n$. By (3.5), (3.7) with $d=m n,(3.4)$, and Lemma 3.1,

$$
\begin{aligned}
n^{-12}\left|g_{A}(\Omega)\right|^{2} & =n^{12}\left(\frac{\Delta\left(\begin{array}{c}
\Omega \\
n
\end{array}\right)}{\Delta\left(\frac{\Omega}{1}\right)}\right)^{2} \\
& =n^{12}\left(n^{-12} \frac{\Delta(\Omega / n)}{\Delta(\Omega)}\right)^{2} \\
& =n^{-12}\left(\frac{\Delta(\sqrt{-m / n})}{\Delta(\sqrt{-m n})}\right)^{2}=c_{m, n}^{-24}
\end{aligned}
$$


is a unit.

Similarly, apply Lemma 3.1 with $I_{1}=[1+\Omega, 2]$ and $I_{2}=[n+\Omega, 2 n]$. Then $A=\left[\begin{array}{cc}1 & 0 \\ (n-1) / 2 & n\end{array}\right]$ and $f=n$. By the same equalities and reasoning as above,

$$
n^{-12}\left|g_{A}\left(\frac{\Omega+1}{2}\right)\right|^{2}=n^{-12}\left(\frac{\Delta\left(\frac{1+\sqrt{-m / n}}{2}\right)}{\Delta\left(\frac{1+\sqrt{-m n}}{2}\right)}\right)^{2}=b_{m, n}^{-24}
$$

is a unit.

Thus, by (3.2), $a_{m, n}^{24}$ is a unit, and so therefore $a_{m, n}$ is also a unit.

The next theorem enables us to explicitly express $a_{m, n}$ in terms of fundamental units.

Theorem 3.3. Let $m$ and $n$ be odd positive integers such that $m n$ is squarefree and $-m n \equiv 3(\bmod 4)$. Set $-4 m n=d_{1} d_{2}$ with $d_{1}>0$ and $d_{i} \equiv 0$ or $1(\bmod 4), i=1,2$. Suppose that $K=Q(\sqrt{-m n})$ has the property that each genus contains exactly one ideal class. Then

$$
a_{m, n}^{h / 4}=\prod \epsilon_{1}^{-w h_{1} h_{2} / w_{2}}
$$

where $h, h_{1}$, and $h_{2}$ are the class numbers of $K, Q\left(\sqrt{d_{1}}\right)$, and $Q\left(\sqrt{d_{2}}\right)$, respectively, $w$ and $w_{2}$ are the numbers of roots of unity in $K$ and $Q\left(\sqrt{d_{2}}\right)$, respectively, $\epsilon_{1}$ is the fundamental unit in $Q\left(\sqrt{d_{1}}\right)$, and the product is over all genus characters $\chi$ associated with the decomposition $-4 m n=d_{1} d_{2}$ such that $\chi([\Omega, n])=\chi([n+\Omega, 2 n])=-1$, and therefore $d_{1}, d_{2}, h_{1}, h_{2}, w_{2}$, and $\epsilon_{1}$ are dependent on $\chi$.

Proof. The proof is very similar to the proof of Theorem 3.1 in [3], and so we provide only a few details.

Let $A_{0}=[\Omega, 1], A_{1}=[\Omega+1,2], A_{2}=[\Omega, n]$, and $A_{3}=[\Omega+n, 2 n]$. For $A=[b+\Omega, a]$ and $z=(b+\Omega) / a$, set

$$
F(A)=\frac{|\eta(z)|^{2}}{\sqrt{a}} .
$$

The number of genus characters such that $\chi\left(A_{2}\right)=\chi\left(A_{3}\right)=-1$ equals $h / 4$. By the same argument that led to eq. (3.3) of [3], we find that

$$
\prod \epsilon_{1}^{w h_{1} h_{2} / w_{2}}=\left(\frac{F\left(A_{2}\right) F\left(A_{3}\right)}{F\left(A_{0}\right) F\left(A_{1}\right)}\right)^{h / 4} .
$$


By (1.15) and (3.10), we see that

$$
\frac{F\left(A_{2}\right) F\left(A_{3}\right)}{F\left(A_{0}\right) F\left(A_{1}\right)}=a_{m, n}^{-1}
$$

Combining (3.11) and (3.12), we complete the proof.

Examples. The class numbers cited below for $|d|<500$ can be found in tables in the texts of Borevich and Shafarevich [5, pp. 422-426], H. Cohen [8, pp. 503-509], and for $0<d<10,000$ in the book of D. A. Buell [7, pp. 224-234]. Lists of fundamental units can be found in [5] (for $d \leq 101$ ), the book by M. Pohst and H. Zassenhaus [11, pp. 432-435] (up to $d \leq 299$ ), and the tables of R. Kortum and G. McNiel [10] (up to $d=10,000$ )

1. Let $m, n=3,7$. Then $h(-84)=4$, and each genus contains one class. The unique character $\chi$ such that $\chi([\Omega, 7])=\chi([\Omega+7,14])=-1$ corresponds to the decomposition $-4 \times 21=12 \times(-7)$. Then $h_{1}=h_{2}=1, \quad w_{2}=w=2$, and $\epsilon_{1}=2+\sqrt{3}$. Thus, by Theorem 3.3,

$$
a_{3,7}=(2+\sqrt{3})^{-1}=2-\sqrt{3}
$$

2. Let $m, n=3,11$. Then $h(-124)=4$. Here $\chi$ corresponds to the decomposition $-124=33 \times(-4)$. Thus, $h_{1}=h_{2}=1, \quad w_{2}=2, \quad w=4, \quad$ and $\epsilon_{1}=23+4 \sqrt{33}$. By Theorem 3.3,

$$
a_{3,11}=(23+4 \sqrt{33})^{-1 / 2}=(23-4 \sqrt{33})^{1 / 2}=2 \sqrt{3}-\sqrt{11}
$$

3. Let $m, n=3,19$. Then $h(-228)=4$. Here $\chi$ corresponds to the decomposition $-228=57 \times(-4)$. Thus $h_{1}=h_{2}=1, w_{2}=2, w=4$, and $\epsilon_{1}=151+20 \sqrt{57}$. Thus, by Theorem 3.3,

$$
a_{3,19}=(151+20 \sqrt{57})^{-1 / 2}=(151-20 \sqrt{57})^{1 / 2}=2 \sqrt{19}-5 \sqrt{3} .
$$

4. Let $m, n=3,31$. Then $h(-372)=4$. Here $\chi$ corresponds to the decomposition $-372=12 \times(-31)$. Thus $h_{1}=1, h_{2}=3, w_{2}=w=2$, and $\epsilon_{1}=2+\sqrt{3}$. Thus, by Theorem 3.3,

$$
a_{3,31}=(2+\sqrt{3})^{-3}=(2-\sqrt{3})^{3}
$$

5. Let $m, n=3,59$. Then $h(-708)=4$. Here $\chi$ corresponds to the decomposition $-708=177 \times(-4)$. Then $h_{1}=h_{2}=1, \quad w_{2}=4, w=2$, and $\epsilon_{1}=62423+4692 \sqrt{177}$. Thus, by Theorem 3.3,

$$
a_{3,59}=(62423+4692 \sqrt{177})^{-1 / 2}=(62423-4692 \sqrt{177})^{1 / 2}=102 \sqrt{3}-23 \sqrt{59} .
$$


6. Let $m, n=5,17$. Then $h(-340)=4$. Here $\chi$ corresponds to the decomposition $-340=17 \times(-20)$. Thus $h_{1}=1, h_{2}=2, w_{2}=w=2$, and $\epsilon_{1}=\sqrt{17}+4$. Thus, by Theorem 3.3,

$$
a_{5,17}=(\sqrt{17}+4)^{-2}=(\sqrt{17}-4)^{2}
$$

Theorem 3.4. Let $m$ and $n$ be distinct primes such that each is congruent to -1 modulo 8. Set $-4 m n=d_{1} d_{2}$ with $d_{1}>0$ and $d_{i} \equiv 0$ or $1(\bmod 4), i=1,2$. Suppose that the class number of $K=Q(\sqrt{-m n})$ equals 8 . Then

$$
a_{m, n}^{2}=\prod \epsilon_{1}^{-w h_{1} h_{2} / w_{2}},
$$

where $h_{1}$ and $h_{2}$ are the class numbers of $Q\left(\sqrt{d_{1}}\right)$ and $Q\left(\sqrt{d_{2}}\right)$, respectively, $w$ and $w_{2}$ are the numbers of roots of unity in $K$ and $Q\left(\sqrt{d_{2}}\right)$, respectively, $\epsilon_{1}$ is the fundamental unit in $Q\left(\sqrt{d_{1}}\right)$, and the product is over all genus characters $\chi$ associated with the decomposition $-4 m n=d_{1} d_{2}$ such that $\chi([\Omega, n])=-1$, and therefore $d_{1}, d_{2}, h_{1}, h_{2}, w_{2}$, and $\epsilon_{1}$ are dependent on $\chi$.

Proof. Since $\left(\frac{2}{m}\right)=\left(\frac{2}{n}\right)=1,[\Omega+1,2]$ is in the principal genus, and $[\Omega, n]$ and $[\Omega+n, 2 n]$ are in the same genus. Let $A_{0}=[\Omega, 1], A_{1}=[\Omega+1,2], A_{2}=[\Omega, n]$, and $A_{3}=[\Omega+n, 2 n]$. Let $F(A)$ be defined by (3.10). Then by identically the same argument as we used in the proof of Theorem 5.2 of [3], but now with 7 there replaced by $n$ here, we conclude that

$$
\prod \epsilon_{1}^{w h_{1} h_{2} / w_{2}}=\left(\frac{F\left(A_{2}\right) F\left(A_{3}\right)}{F\left(A_{0}\right) F\left(A_{1}\right)}\right)^{2}
$$

We now complete the proof by using the same argument that we used in the proof of Theorem 3.3.

The following two examples are not found in Ramanujan's work.

1. Let $m, n=7,31$. Then $h(-868)=8$, and $w=2$. There are $8 / 4=2$ genus characters $\chi$ such that $\chi([\sqrt{-217}, 31])=-1$, and they correspond to the decompositions $-868=217 \times(-4)=124 \times(-7)$. Also, $h_{1}=h_{2}=1, w_{2}=4$, and $\epsilon_{1}=3844063+260952 \sqrt{217} ; \quad h_{1}=h_{2}=1, \quad w_{2}=2$, and $\epsilon_{1}=1520+273 \sqrt{31}$, respectively. (See Theorem 5.3 of [3].) Thus, by Theorem 3.4,

$$
\begin{aligned}
a_{7,31} & =(3844063+260952 \sqrt{217})^{-1 / 4}(1520+273 \sqrt{31})^{-1 / 2} \\
& =(524-249 \sqrt{31})^{1 / 2}(1520-273 \sqrt{31})^{1 / 2}
\end{aligned}
$$


2. Let $m, n=7,79$. Then $h=8$ and $w=2$. The two genus characters $\chi$ such that $\chi([\sqrt{-2212}, 79])=-1$ correspond to the decompositions $-2212=28 \times(-79)=$ $553 \times(-4)$. Then $h_{1}=1, h_{2}=5, w_{2}=2$, and $\epsilon_{1}=8+3 \sqrt{7} ; h_{1}=h_{2}=1, w_{2}=4$, and $\epsilon_{1}=624,635,837,407+26,562,217,704 \sqrt{553}$, respectively. Thus, by Theorem 3.4 ,

$$
\begin{aligned}
a_{7,79} & =(8+3 \sqrt{7})^{-5 / 2}(624,635,837,407+26,562,217,704 \sqrt{553})^{-1 / 4} \\
& =(514,088-194,307 \sqrt{7})^{1 / 2}(211,227 \sqrt{7}-62,876 \sqrt{79})^{1 / 2} .
\end{aligned}
$$

We establish one further theorem about units.

Theorem 3.5. Let $m$ and $n$.be odd positive integers such that $m n$ is squarefree and $-m n \equiv 1(\bmod 8)$. Then $a_{m, n}$ is a unit.

Proof. With the definition (1.3), it is a straightforward exercise to show that, for $y>0$,

$$
\left|\frac{\eta\left(\frac{1+i y}{4}\right)}{\eta\left(\frac{1+i y}{2}\right)}\right|^{2}=\left|\frac{\eta\left(\frac{1+i y}{2}\right)}{\eta(i y)}\right| .
$$

Thus, by (1.15),

$$
a_{m, n}^{12}=n^{12}\left|\frac{\eta^{96}\left(\frac{1+\sqrt{-m n}}{2}\right) \eta^{48}\left(\frac{1+\sqrt{-m / n}}{4}\right)}{\eta^{96}\left(\frac{1+\sqrt{-m / n}}{2}\right) \eta^{48}\left(\frac{1+\sqrt{-m n}}{4}\right)}\right|
$$

If we define

$$
c_{m, n}^{\prime}=\sqrt{n}\left|\frac{\eta^{2}\left(\frac{1+\sqrt{-m n}}{4}\right)}{\eta^{2}\left(\frac{1+\sqrt{-m / n}}{4}\right)}\right|,
$$

then, by (3.13), (3.2), and (3.4),

$$
a_{m, n}^{12}=\frac{b_{m, n}^{48}}{c_{m, n}^{24}}
$$

We now use Lemma 3.1 and proceed as in the proof of Theorem 3.2. Letting 
$I_{1}=[\Omega, 1]$ and $I_{2}=[\Omega, n]$, where $\Omega=(1+\sqrt{-m n}) / 2$, we deduce that $b_{m, n}^{24}$ is a unit. Letting $I_{1}=[\Omega, 2]$ and $I_{2}=[\Omega+n, 2 n]$, we deduce that $c_{m, n}^{\prime 24}$ is a unit. Thus, the theorem now follows from (3.14).

As an example, let $m, n=3,5$. Then, by Theorem 3.5, $a_{3,5}$ is a unit. By using Kronecker's limit formula to calculate $b_{3,5}$ and $c_{3,5}^{\prime}$, we can show that $a_{3,5}=(3-\sqrt{5}) / 2$. We omit the details.

4. $\varphi\left(e^{-m \pi}\right) / \varphi\left(e^{-\pi}\right)$ is an algebraic integer

We shall use the following lemma from Stark's paper [16, Lemma 15]. Recall that $g_{A}$ is defined by (3.8).

Lemma 4.1. If $\omega$ is in a complex quadratic field, $\operatorname{Im} \omega>0$, and $A \in S_{1}(f)$, then $g_{A}(\omega)$ is an algebraic integer which divides $f^{12}$.

We need two lemmas for the proof of the main theorem of this section, Theorem 4.4.

Lemma 4.2. If $m$ is a positive integer, then $\Delta\left(\frac{1+m i}{2}\right) / \Delta(1+m i)$ is an algebraic integer dividing $2^{12}$.

Proof. By (3.5),

$$
\frac{\Delta\left(\frac{1+m i}{2}\right)}{\Delta(1+m i)}=\frac{2^{12} \Delta\left(\begin{array}{c}
1+m i \\
2
\end{array}\right)}{\Delta\left(\begin{array}{c}
1+m i \\
1
\end{array}\right)}=\frac{2^{12} \Delta\left(\left(\begin{array}{ll}
1 & 0 \\
0 & 2
\end{array}\right)\left(\begin{array}{c}
1+m i \\
1
\end{array}\right)\right)}{\Delta\left(\begin{array}{c}
1+m i \\
1
\end{array}\right)}=g_{A}(1+m i),
$$

where $A=\left(\begin{array}{ll}1 & 0 \\ 0 & 2\end{array}\right) \in S_{1}(2)$. The desired result now clearly follows from Lemma 4.1 .

Remark. It follows from Lemma 4.2 that

$$
\frac{\Delta\left(\frac{1+m i}{2}\right)}{\Delta(1+m i)} \cdot M=2^{12},
$$

where

$$
M=2^{12} \cdot \frac{\Delta(1+m i)}{\Delta\left(\frac{1+m i}{2}\right)}
$$

is an algebraic integer dividing $2^{12}$. 
Lemma 4.3. Let $m, n$ denote a pair of positive integers, and set

$$
\beta_{m, n}=\frac{\Delta\left(\frac{1+m n i}{2}\right)}{\Delta\left(\frac{1+n i}{2}\right)}
$$

Then $m^{12} \beta_{m, n}$ is an algebraic integer dividing $m^{12}$ if $m$ is odd, and $(2 m)^{12} \beta_{m, n}$ is an algebraic integer dividing $(4 m)^{12}$ if $m$ is even.

Proof. First assume that $m$ is odd. Then

$$
\begin{aligned}
m^{12} \beta_{m, n} & =\frac{m^{12} \Delta\left(\frac{1+m n i}{2}\right)}{\Delta\left(\frac{1+n i}{2}\right)}=\frac{m^{12} 2^{12} \Delta\left(\begin{array}{c}
1+m n i \\
2
\end{array}\right)}{2^{12} \Delta\left(\begin{array}{c}
1+n i \\
2
\end{array}\right)} \\
& =\frac{m^{12} \Delta\left(\left(\begin{array}{cc}
m & (1-m) / 2 \\
0 & 1
\end{array}\right)\left(\begin{array}{c}
1+n i \\
2
\end{array}\right)\right)}{\Delta\left(\begin{array}{c}
1+n i \\
2
\end{array}\right)}=g_{A}\left(\frac{1+n i}{2}\right)
\end{aligned}
$$

where $A=\left(\begin{array}{cc}m & (1-m) / 2 \\ 0 & 1\end{array}\right) \in S_{1}(m)$, as $(1-m) / 2$ is integral.

In the case that $m$ is even,

$$
\begin{aligned}
(2 m)^{12} \beta_{m, n} & =\frac{(2 m)^{12} \Delta\left(\frac{1+m n i}{2}\right)}{\Delta\left(\frac{1+n i}{2}\right)}=\frac{(2 m)^{12} 4^{12} \Delta\left(\begin{array}{c}
2+2 m n i \\
4
\end{array}\right)}{2^{12} \Delta\left(\begin{array}{c}
1+n i \\
2
\end{array}\right)} \\
& =\frac{(4 m)^{12} \Delta\left(\left(\begin{array}{cc}
2 m & 1-m \\
0 & 2
\end{array}\right)\left(\begin{array}{c}
1+n i \\
2
\end{array}\right)\right)}{\Delta\left(\begin{array}{c}
1+n i \\
2
\end{array}\right)}=g_{A}\left(\frac{1+n i}{2}\right)
\end{aligned}
$$

where $A=\left(\begin{array}{cc}2 m & 1-m \\ 0 & 2\end{array}\right) \in S_{1}(4 m)$, because $(2 m, 1-m, 2)=1$, as $1-m$ is odd.

By Lemma 4.1, the proof is complete in both cases.

Theorem 4.4. Let $m$ and $n$ be positive integers. Then $\varphi\left(e^{-m n \pi}\right) / \varphi\left(e^{-n \pi}\right)$ is algebraic. Furthermore, if $m$ is odd, then $\sqrt{2 m} \varphi\left(e^{-m n \pi}\right) / \varphi\left(e^{-n \pi}\right)$ is an algebraic integer dividing $2 \sqrt{m}$, while if $m$ is even, then $2 \sqrt{m} \varphi\left(e^{-m n \pi}\right) / \varphi\left(e^{-n \pi}\right)$ is an algebraic integer dividing $4 \sqrt{m}$. 
Proof. Let $q=\exp (\pi i \tau)$, where $\operatorname{Im} \tau>0$. From (1.1) and (1.3),

$$
\varphi(q)=\frac{(-q ;-q)_{\infty}^{2}}{\left(q^{2} ; q^{2}\right)_{\infty}}=\frac{\eta^{2}\left(\frac{\tau+1}{2}\right)}{\eta(\tau+1)}
$$

We can thus write $\varphi\left(e^{-m n \pi}\right) / \varphi\left(e^{-n \pi}\right)$ in the form

$$
\begin{aligned}
\frac{\varphi\left(e^{-m n \pi}\right)}{\varphi\left(e^{-m \pi}\right)} & =\frac{\eta^{2}\left(\frac{1+m n i}{2}\right)}{\eta(1+m n i)} / \frac{\eta^{2}\left(\frac{1+n i}{2}\right)}{\eta(1+n i)} \\
& =\frac{\eta\left(\frac{1+m n i}{2}\right)}{\eta(1+m n i)} \cdot \frac{\eta(1+n i)}{\eta\left(\frac{1+n i}{2}\right)} \cdot \frac{\eta\left(\frac{1+m n i}{2}\right)}{\eta\left(\frac{1+n i}{2}\right)} .
\end{aligned}
$$

If $m$ is odd, then

$$
\frac{(2 m)^{12} \varphi^{24}\left(e^{-m n \pi}\right)}{\varphi^{24}\left(e^{-n \pi}\right)}=\frac{\Delta\left(\frac{1+m n i}{2}\right)}{\Delta(1+m n i)} \cdot \frac{2^{12} \Delta(1+n i)}{\Delta\left(\frac{1+n i}{2}\right)} \cdot \frac{m^{12} \Delta\left(\frac{1+m n i}{2}\right)}{\Delta\left(\frac{1+n i}{2}\right)}
$$

is an algebraic integer dividing $2^{12} \cdot 2^{12} \cdot \mathrm{m}^{12}=2^{24} \mathrm{~m}^{12}$, by Lemma 4.2 , the remark prior to Lemma 4.3, and Lemma 4.3. The first part of the conclusion of Theorem 4.4 now follows.

If $m$ is even, then

$$
\frac{(4 m)^{12} \varphi^{24}\left(e^{-m n \pi}\right)}{\varphi^{24}\left(e^{-n \pi}\right)}=\frac{\Delta\left(\frac{1+m n i}{2}\right)}{\Delta(1+m n i)} \cdot \frac{2^{12} \Delta(1+n i)}{\Delta\left(\frac{1+n i}{2}\right)} \cdot \frac{(2 m)^{12} \Delta\left(\frac{1+m n i}{2}\right)}{\Delta\left(\frac{1+n i}{2}\right)}
$$

is an algebraic integer dividing $2^{12} \cdot 2^{12} \cdot(4 m)^{12}=2^{24}(4 m)^{12}$, by Lemma 4.2 , the remark prior to Lemma 4.3, and Lemma 4.3. The second part of the conclusion of Theorem 4.4 now follows.

Corollary 4.5. Let $m$ be a positive integer. Then $\varphi\left(e^{-m x}\right) / \varphi\left(e^{-\pi}\right)$ is algebraic. Furthermore, if $m$ is odd, then $\sqrt{2 m} \varphi\left(e^{-m \pi}\right) / \varphi\left(e^{-\pi}\right)$ is an algebraic integer dividing $2 \sqrt{m}$, while if $m$ is even, then $2 \sqrt{m} \varphi\left(e^{-m \pi}\right) / \varphi\left(e^{-\pi}\right)$ is an algebraic integer dividing $4 \sqrt{m}$.

Proof. Set $n=1$ in Theorem 4.4 . 


\section{REFERENCES}

1. B. C. Berndt, Ramanujan's Notebooks, Part III (Springer-Verlag, New York, 1991).

2. B. C. Berndt and H. H. Chan, Ramanujan's explicit values for the classical thetafunction, Mathematika 42 (1995), 278-294.

3. B. C. Berndt, H. H. Chan and L.-C. Zhang, Ramanujan's class invariants, Kronecker's limit formula, and modular equations, Trans. Amer. Math. Soc., to appear.

4. B. C. Berndt, H. H. Chan and L.-C. Zhang, Explicit evaluations of the RogersRamanujan continued fraction 480 (1996), 141-159.

5. Z. I. Borevich and I. R. Shafarevich, Number Theory (Academic Press, New York, 1966).

6. J. M. BoRWeIn and P. B. Borwern, Pi and the AGM (Wiley, New York, 1987).

7. D. A. Buell, Binary Quadratic Forms, Classical Theory and Modern Computations (Springer-Verlag, New York, 1989).

8. H. CoHEN, A Course in Computational Algebraic Number Theory (Springer-Verlag, Berlin, 1993).

9. D. A. Cox, Primes of the Form $x^{2}+n y^{2}$ (Wiley, New York, 1989).

10. R. Kortum AND G. MCNiel, A Table of Periodic Continued Fractions (Lockheed Aircraft Corporation, Sunnyvale, CA).

11. M. Pohst and H. ZASSEnhaus, Algorithmic Algebraic Number Theory (Cambridge University Press, Cambridge, 1989).

12. K. G. Ramanathan, Some applications of Kronecker's limit formula, J. Indian Math. Soc. 52 (1987), 71-89.

13. S. Ramanujan, Modular equations and approximations to $\pi$, Quart. J. Math. (Oxford) 45 (1914), 350-372.

14. S. Ramanujan, Notebooks (2 volumes) (Tata Institute of Fundamental Research, Bombay, 1957).

15. S. Ramanujan, Collected Papers (Chelsea, New York, 1962).

$16 \mathrm{H}$. M. STARK, Values of $L$-functions at $s=1 \mathrm{I}$. $L$-functions for quadratic forms, $A d v$. Math. 7 (1971), 301-343.

17. H. WeBER, Lehrbuch der Algebra, dritter Band (Chelsea, New York, 1961).

DepartMent OF Mathematics

1409 WEST GREEN STREET

UNIVERSITY OF ILLINOIS

URBANA, IL 61801

U.S.A.

Department of Mathematics

National Chung Cheng University

MinhsiUNG

CHIAYI 621

TAIWAN
Department of Mathematics

SOUTHWEST MISSOURI STATE UNIVERSITY

SPRINGFIELD, MO 65804

U.S.A. 